\title{
DIGITAL REPRESENTATIONS USING THE GREATEST INTEGER FUNCTION
}

\author{
BRUCE REZNICK
}

\begin{abstract}
Let $S_{d}(\alpha)$ denote the set of all integers which can be expressed in the form $\sum \varepsilon_{i}\left[\alpha^{i}\right]$, with $\varepsilon_{i} \in\{0, \ldots, d-1\}$, where $d \geq 2$ is an integer and $\alpha \geq 1$ is real, and let $I_{d}$ denote the set of $\alpha$ so that $S_{d}(\alpha)=\mathbf{Z}^{+}$. We show that $I_{d}=\left[1, r_{d}\right) \cup\{d\}$, where $r_{2}=13^{1 / 4}, r_{3}=22^{1 / 3}$ and $r_{4}=\left(d^{2}-d-2\right)^{1 / 2}$ for $d \geq 4$. If $\alpha \notin I_{d}$, we show that $T_{d}(\alpha)$, the complement of $S_{d}(\alpha)$, is infinite, and discuss the density of $T_{d}(\alpha)$ when $\alpha<d$. For $d \geq 4$ and a particular quadratic irrational $\beta=\beta(d)<d$, we describe $T_{d}(\beta)$ explicitly and show that $\left|T_{d}(\beta) \cap[0, n]\right|$ is of order $n^{e(d)}$, where $e(d)<1$.
\end{abstract}

\section{INTRODUCTION}

In the usual base $d$ digital representation, every nonnegative integer $m$ is written (uniquely) in the form $m=\sum \varepsilon_{i} d^{i}$, where $\varepsilon_{i} \in[[0, d-1]]$. (Here and throughout the paper, we shall denote $[a, b] \cap \mathbf{Z}$ by $[[a, b]]$.) This remarkable fact has inspired many generalizations, especially when $d=2$. (The survey papers [4] and [8] together contain sixty references.) In this paper, we replace $d^{i}$ by $\left[\alpha^{i}\right]$, where $[x]$ denotes the greatest integer $\leq x$ and $\alpha \geq 1$ is a fixed real number, and allow the value of $d$ for the range of $\varepsilon_{i}$ to become a second parameter.

The fine behavior of the sequence $\left[\alpha^{i}\right]$ has also attracted much interest, especially in the equivalent form $\left\{\alpha^{i}\right\}=\alpha^{i}-\left[\alpha^{i}\right]$. For example, Mahler has asked whether there exists $\lambda$ so that $\left\{\lambda(3 / 2)^{n}\right\}$ is equidistributed on $[0,1)$. The only nonintegral $\alpha$ 's for which $\left\{\alpha^{i}\right\}$ is well understood appear to be the PV numbers (algebraic integers whose conjugates lie in $|z|<1$ ). A representation $m=\sum \varepsilon_{i}\left[\alpha^{i}\right]$ can also be viewed as a partition of $m$ into the set $\left(\left[\alpha^{0}\right],\left[\alpha^{1}\right],\left[\alpha^{2}\right], \ldots\right)$ with at most $d-1$ repetitions; this question has previously been studied when $\alpha<d$ is an integer.

Received by the editors November 9,1987 and, in revised form, July $25,1988$.

1980 Mathematics Subject Classification (1985 Revision). Primary 11A63; Secondary 05A15.

Author supported in part by the Alfred P. Sloan Foundation and the National Science Foundation. 
We now introduce our most important definitions. Let

$$
\begin{gathered}
S_{d}(\alpha)=\left\{\sum_{i=0}^{T} \varepsilon_{i}\left[\alpha^{i}\right]: \varepsilon_{i} \in[[0, d-1]], T \geq 0\right\}, \\
T_{d}(\alpha)=\mathbf{Z}^{+} \backslash S_{d}(\alpha), \\
I_{d}=\left\{\alpha: S_{d}(\alpha)=\mathbf{Z}^{+}\right\} .
\end{gathered}
$$

In this paper we compute $I_{d}$ and discuss the cardinality and density of $T_{d}(\alpha)$ when $\alpha \notin I_{d}$. We give an explicit description of $T_{d}(\beta)$ for a family of pairs $(d, \beta)$ in which $\beta=\beta(d)$ is a certain PV number.

If $\alpha>d$, then an easy counting argument (see $\S 3$ ) shows that $S_{d}(\alpha)$ has density 0 , so $\alpha \notin I_{d}$. If $\alpha \leq d$ is an integer, then the base $\alpha$ digital representations are included in (1.1), so $\alpha \in I_{d}$. One might expect this argument to generalize to nonintegral $\alpha<d$ by some version of the pigeonhole principle, so that our problem is trivial. This is not true. Suppose $d \geq 3$ and $\alpha \in\left[\left(d^{2}-1\right)^{1 / 2}, d\right)$, so $[\alpha]=d-1$ and $\left[\alpha^{2}\right]=d^{2}-1$, and suppose $d^{2}-d+1 \leq m \leq d^{2}-2$. If $m=\sum \varepsilon_{i}\left[\alpha^{i}\right]$, then $\varepsilon_{i}=0$ for $i \geq 2$ as $m<\left[\alpha^{2}\right]$. But then $m \leq(d-1)\left(\left[\alpha^{0}\right]+\left[\alpha^{1}\right]\right)=d^{2}-d$, a contradiction. A similar argument works for $d=2$, with $m=6$ and $\alpha \in\left[7^{1 / 3}, 2\right)$, by considering $\varepsilon_{0}, \varepsilon_{1}$ and $\varepsilon_{2}$.

This paper contains five theorems (and one conjecture) about $S_{d}(\alpha), T_{d}(\alpha)$ and $I_{d}$.

\section{Theorem A.}

(i) $I_{2}=\left[1,13^{1 / 4}\right) \cup\{2\}$,

(ii) $I_{3}=\left[1,22^{1 / 3}\right) \cup\{3\}$,

(iii) $I_{d}=\left[1,\left(d^{2}-d+2\right)^{1 / 2}\right) \cup\{d\}, d \geq 4$.

The description of $I_{2}$ may be deduced from [7, Theorem 5]. Contrary to appearances, it is not true that $m \in S_{d}(\alpha)$ implies $m \in S_{d}(\gamma)$ for $\gamma \in[1, \alpha)$. We must prove Theorem $\mathrm{A}$ by an appeal to ten separate cases.

Theorem B. If $\alpha \notin I_{d}$, then $\left|T_{d}(\alpha)\right|=\infty$.

When $d=2$, this may be deduced from [7, Theorem 3]. Theorem B is proved by the inductive construction of an infinite sequence in $T_{d}(\alpha)$. The construction follows [7], where the idea is attributed to J. Folkman.

Theorem C. If $\alpha<d$ and $\alpha \notin I_{d}$, then there exists a positive integer $t=t(\alpha, d)$ so that every integer $m \geq 0$ can be written as $\sum \varepsilon_{i}\left[\alpha^{i}\right]$, with $\varepsilon_{i} \in[[0, d]]$ for $i \leq t$ and $\varepsilon_{i} \in[[0, d-1]]$ for $i>t$.

Theorem D. If $\alpha<d$ and $\alpha \notin I_{d}$, then $S_{d}(\alpha)$ has positive density.

Theorems C and D are immediate consequences of the analysis of the "greedy" representations of $m$ as $\sum \varepsilon_{i}\left[\alpha^{i}\right]$. In Theorem $\mathrm{D}$, one would expect $S_{d}(\alpha)$ to have density one; Theorem $\mathrm{E}$ proves this in one special case. 
Theorem E. Suppose $d \geq 4$ and $\beta=\beta(d)=\frac{1}{2}\left(d-1+\left(d^{2}+2 d-3\right)^{1 / 2}\right)$. Then $\beta \notin I_{d}$ and $S_{d}(\beta)$ has density 1 .

The proof of Theorem E hinges on the fact that $\beta$ is a PV number, and so the behavior of $\left[\beta^{i}\right]$ is intimately related to the behavior of the sequence $\beta^{r}+\bar{\beta}^{r}$, which satisfies a second-order linear recurrence by Newton's identity. We can describe the workings of the greedy representation for $(d, \beta)$ in exact detail and are able to give an explicit description of the set $T_{d}(\beta)$. We show that $N_{r}=\sharp\left\{m \in T_{d}(\beta): m \leq\left[\beta^{r+1}\right]-1\right\}$ satisfies a fourth-order linear recurrence, and $N_{r} \approx c_{d} \gamma^{r}$ asymptotically, where $\gamma=\gamma(d)<\beta(d)$ is another quadratic irrational. Thus $S_{d}(\beta)$ has density 1 .

Conjecture F. If $\alpha<d$ and $\alpha \notin I_{d}$, then $S_{d}(\alpha)$ has density 1 .

It seems unlikely that these arguments of Theorem $E$ can be generalized sufficiently to prove Conjecture $\mathrm{F}$, especially for transcendental $\alpha$. A counterintuitive bit of negative evidence is this: if $d \geq 2$ and $r \geq 2$, then there exists an open interval $I_{r, d} \subset[1, d] \backslash I_{d}$ so that $d^{r-1}-d^{r-2}$ integers $\leq d^{r}$ lie in $T_{d}(\alpha)$ for $\alpha \in I_{r, d}$. This result does not violate Conjecture $\mathrm{F}$, since any fixed $\alpha \in[1, d] \backslash I_{d}$ only belongs to finitely many $I_{r, d}$. Nevertheless, it seems unlikely that a uniformity argument can be used to prove the conjecture.

The paper is organized as follows. $\S 2$ presents the literature on a related question: complete and entirely complete sequences, and gives Brown's criterion, which is essential to the sequel. In $\S 3-\S 6$, we prove Theorems $A$ through $\mathrm{E}$, and in $\S 7$, we discuss Conjecture $\mathrm{F}$ and the related partition problems.

Most of the results of this paper were found while the author was a Visiting Associate at the California Institute of Technology in early 1985. The author thanks the Caltech faculty and staff for their hospitality during his visit.

\section{Preliminaries}

Let $A=\left(a_{0}, a_{1}, \ldots\right)$ be a nondecreasing sequence of positive integers and, for $d \geq 2$, define the sets

$$
S_{d}^{n}(A)=\left\{\sum_{i=0}^{n} \varepsilon_{i} a_{i}: \varepsilon_{i} \in[[0, d-1]]\right\},
$$

so that, (cf. (1.1)) $S_{d}(A)=\bigcup_{n=0}^{\infty} S_{d}^{n}(A)$. The sequence $A$ is called $d$-complete if $S_{d}(A)$ contains all sufficiently large integers and entirely $d$-complete if $S_{d}(A)=$ $\mathbf{Z}^{+}$. In this terminology, $\alpha \in I_{d}$ precisely when the sequence $\left(\left[\alpha^{i}\right]\right)$ is entirely $d$-complete. When $d=2$, one speaks of complete and entirely complete sequences; this was the first case discussed in the literature. Hoggatt and King $([10,11])$ posed the following problem about the Fibonacci sequence $F=(1,1,2,3,5, \ldots)$ : Let $F^{\prime}$ denote $F$ with any one element deleted and $F^{\prime \prime}$ denote $F$ with any two elements deleted. Show that every $F^{\prime}$ (and no $F^{\prime \prime}$ ) is entirely complete. Brown [2] in 1960 generalized the solution to this problem, and gave a necessary and sufficient condition (Lemma 2.3 below) for a sequence 
to be entirely complete. Alder [1] in 1962 and Fridy [5] in 1965 independently gave the definitions for $d \geq 3$ and generalized Brown's criterion.

Given a sequence $A$, let

$$
A^{d}=\left(a_{0}, \ldots, a_{0}, a_{1}, \ldots, a_{1}, a_{2}, \ldots\right),
$$

where each $a_{i}$ from $A$ occurs $d-1$ times in $A^{d}$. It is easy to see that the $d$-(entire)-completeness of $A$ is equivalent to the (entire)-completeness of $A^{d}$. In 1964, Graham [7] considered the two-parameter family of sequences $A_{t}(\alpha)=$ $\left([t \alpha],\left[t \alpha^{2}\right],\left[t \alpha^{3}\right], \ldots\right)$ with $0<t$ and $1<\alpha<2$. Erdös had conjectured that $A_{t}(\alpha)$ is always complete, even if not entirely complete. Graham proved that, when $t<1$, completeness implies entire completeness and determined the set of $(t, \alpha)$ for which $A_{t}(\alpha)$ is complete. Specializations of Theorems 5 and 3 in [7] to $t=\alpha^{-1}$ give the relevant portions $(d=2)$ of Theorems A and $\mathbf{B}$.

We now give Brown's criterion and its proof, which is short and illuminating.

Lemma 2.3 (Brown). Let $A=\left(a_{0}, a_{1}, \ldots\right)$ be a nondecreasing sequence of positive integers, and let $s_{k}=\sum_{i=0}^{k} a_{i}$. Then $A$ is entirely complete if and only if $a_{0}=1$ and

$$
a_{r} \leq s_{r-1}+1, r \geq 1 \text {. }
$$

If (2.4) is not satisfied and $m \in\left[\left[s_{r-1}+1, a_{r}-1\right]\right]$, then $m \notin S_{2}(A)$.

Proof. The smallest integer in $S_{2}(A)$ is $a_{0}$, so $a_{0}=1$ is both necessary and sufficient for 1 to belong to $S_{2}(A)$. If $A$ is entirely complete, then for all $r \geq 1$, $a_{r}-1 \in S_{2}(A)$; that is, $a_{r}-1=\sum \varepsilon_{i} a_{i}, \varepsilon_{i} \in\{0,1\}$. As $a_{i} \geq a_{r}$ for $i \geq r$, it follows that $\varepsilon_{i}=0$ for $i \geq r$. Thus,

$$
a_{r}-1=\sum_{i=0}^{r-1} \varepsilon_{i} a_{i} \leq \sum_{i=0}^{r-1} a_{i}=s_{r-1}
$$

which is (2.4). This argument also shows that, if (2.4) fails and $m$ is in the indicated range, then $m \notin S_{2}(A)$. We shall use this reasoning repeatedly.

Now suppose that $a_{0}=1$ and (2.4) holds. We prove by induction that $S_{2}^{n}(A)=\left[\left[0, S_{n}\right]\right]$. This is clear for $n=0$; suppose it is true for $n=r-1$. Then by distinguishing the cases $\varepsilon_{r}=0$ and 1 in (2.1), we see that

$$
S_{2}^{r}(A)=S_{2}^{r-1}(A) \cup\left(S_{2}^{r-1}(A)+a_{r}\right)=\left[\left[0, s_{r-1}\right]\right] \cup\left[\left[a_{r}, s_{r}\right]\right] .
$$

By (2.4), the two sets of integers merge without gap, completing the inductive step. Finally, $a_{j} \geq a_{0} \geq 1$, so $m \in S_{2}^{m-1}(A) \subseteq S_{2}(A)$.

Lemma 2.7 (Alder). $A$ nondecreasing sequence of positive integers, $B=$ $\left(b_{0}, b_{1}, \ldots\right)$, is entirely $d$-complete if and only if $b_{0}=1$ and, for all $s \geq 1$,

$$
b_{s} \leq 1+(d-1) \sum_{i=0}^{s-1} b_{i} \text {. }
$$


If (2.8) fails and $1+(d-1) \sum_{i=0}^{s-1} b_{i} \leq m \leq b_{s}-1$, then $m \notin S_{d}(B)$.

Proof. Apply Lemma 2.3 to $A=B^{d}$. If $a_{r}=a_{r-1}$, then (2.4) is automatic. If $a_{r}>a_{r-1}$, then $r=(d-1) s, a_{(d-1) s}=b_{s}$ and $a_{(d-1) s-1}=b_{s-1}$, so (2.4) reduces to (2.8).

Henceforth, we shall abbreviate $\left[\alpha^{i}\right]$ and $\left[\beta^{i}\right]$ by $\alpha_{i}$ and $\beta_{i}$ respectively, and write $\underline{\alpha}=\left(\alpha_{0}, \alpha_{1}, \ldots\right)$ and $\underline{\beta}=\left(\beta_{0}, \beta_{1}, \ldots\right)$. We now apply Lemma 2.7 to our situation. Let

$$
\varphi(\alpha, d, k)=1+(d-1) \sum_{i=0}^{k-1} \alpha_{i}-\alpha_{k}=\sum_{i=0}^{k-1}\left(d \alpha_{i}-\alpha_{i+1}\right)
$$

Since $\alpha_{0}=1$ for all $\alpha$, Lemma 2.7 states that $\alpha \in I_{d}$ if and only if $\varphi(\alpha, d, k)$ $\geq 0$ for all $k \geq 1$. We say that $(\alpha, d, k)$ is bad if $\varphi(\alpha, d, k) \leq-1$ and $d \alpha_{k-1}-\alpha_{k} \leq-1$. By looking at the smallest $k$ for which $\varphi(\alpha, d, k)<0$, we see that Lemma 2.7 gives the following criterion for $I_{d}$ :

Lemma 2.10. $\alpha \notin I_{d}$ if and only if $(\alpha, d, k)$ is bad for some $k$.

Since $\alpha^{i}-1<\alpha_{i} \leq \alpha^{i}, \alpha_{i}$ is "close" to $\alpha \cdot \alpha_{i-1}$; when $d>\alpha$, this leads to a useful inequality which bounds the value of $k$ in a bad triple.

Lemma 2.11. If $1<\alpha<d$ and $d \alpha_{k-1}-\alpha_{k} \leq-1$, then

$$
\alpha_{k-1}<(\alpha-1) /(d-\alpha) \text {. }
$$

In particular, if $k \geq 2$, then

$$
\begin{gathered}
\left(\alpha^{k-2}+\cdots+\alpha+1\right)<1 /(d-\alpha), \\
\alpha^{k-1}<(d-1) /(d-\alpha) .
\end{gathered}
$$

Proof. By hypothesis,

$$
d \alpha_{k-1} \leq \alpha_{k}-1 \leq \alpha^{k}-1<\alpha\left(\alpha_{k-1}+1\right)-1,
$$

whence (2.12); (2.13) and (2.14) follow from (2.12) and $\alpha_{k-1}>\alpha^{k-1}-1$.

\section{THE PROOF OF THEOREM A}

We prove Theorem A by dividing it into ten cases (this notation differs harmlessly from that in the introduction). We shall retain this case notation in the proof of Theorem B.

\section{Theorem A.}

(a) If $\alpha>d$, then $\alpha \notin I_{d}$.

(b) If $\alpha \leq d$ and $\alpha \in \mathbf{Z}$, then $\alpha \in I_{d}$.

(c) If $d \geq 3$ and $1 \leq \alpha \leq d-1$, then $\alpha \in I_{d}$.

(d) If $1 \leq \alpha<13^{1 / 4}$, then $\alpha \in I_{2}$.

(e) If $13^{1 / 4} \leq \alpha<7^{1 / 3}$, then $\alpha \notin I_{2}$ and $12 \in T_{2}(\alpha)$.

(f) If $7^{1 / 3} \leq \alpha<2$, then $\alpha \notin I_{2}$ and $6 \in T_{2}(\alpha)$. 
(g) If $2 \leq \alpha<22^{1 / 3}$, then $\alpha \in I_{3}$.

(h) If $22^{1 / 3} \leq \alpha<8^{1 / 2}$, then $\alpha \notin I_{3}$ and $21 \in T_{3}(\alpha)$.

(j) If $d-1 \leq \alpha<\left(d^{2}-d+2\right)^{1 / 2}$ and $d \geq 4$, then $\alpha \in I_{d}$.

(k) If $\left(d^{2}-d+2\right)^{1 / 2} \leq \alpha<d$ and $d \geq 3$, then $\alpha \notin I_{d}$ and $d^{2}-d+1 \in T_{d}(\alpha)$.

The numerical values of the surds are: $13^{1 / 4} \approx 1.89883,7^{1 / 3} \approx 1.91293$, $22^{1 / 3} \approx 2.80204$ and $8^{1 / 2} \approx 2.82843$. The proofs of the first two cases were discussed in the introduction.

Proof of Theorem A(a). As in the proof of Lemma 2.3, if $m \in S_{d}(\alpha)$ and $m \leq \alpha_{n}-1$, then $m=\sum_{i=0}^{n-1} \varepsilon_{i} \alpha_{i} \in S_{d}^{n-1}(\alpha)$. There are only $d^{n}$ formally distinct sums in $S_{d}^{n-1}(\alpha)$, so at most $d^{n}$ of the $\alpha_{n}$ integers in $\left[\left[0, \ldots, \alpha_{n}-1\right]\right]$ lie in $S_{d}(\alpha)$. Since $\alpha>d, \alpha_{n}>\alpha^{n}-1>d^{n}$ for $n$ sufficiently large. As $d^{n} /\left(\alpha^{n}-1\right) \rightarrow 0$, the first part of Theorem $\mathrm{B}$ is also proved.

Proof of Theorem $\mathrm{A}(\mathrm{b})$. Write $m$ in base $\alpha$; as $\alpha_{i}=\alpha^{i}, m=\sum \varepsilon_{i} \alpha_{i}$, with $\varepsilon_{i} \leq \alpha-1 \leq d-1$.

In the remaining eight cases, $\alpha<d$ is not an integer. The strategy of our proof is this: we shall either present a bad triple $(\alpha, d, k)$, showing that $\alpha \notin$ $I_{d}$, or use Lemma 2.11 to show that no bad triple exists.

Lemma 3.1. Suppose $1 \leq \alpha<d$. Then $\varphi(\alpha, d, 1) \geq 0$, and $\varphi(\alpha, d, 2) \geq 0$ if and only if $1 \leq \alpha<\left(d^{2}-d+2\right)^{1 / 2}$. Thus, if $\alpha<\left(d^{2}-d+2\right)^{1 / 2}$ and $(\alpha, d, k)$ is bad, then $k \geq 3$ and $(d-\alpha) \alpha^{k-1}<d-1$.

Proof. Write $\alpha_{1}=s$ and $\alpha_{2}=s^{2}+u$, so $1 \leq s \leq d-1$ and $0 \leq u \leq 2 s$. Then $\varphi(\alpha, d, 1)=d-s \geq 0$ and

$$
\varphi(\alpha, d, 2)=d+(d-1) s-\left(s^{2}+u\right)=(d-s)(1+s)-u .
$$

If $s \leq d-2$, then $\varphi(\alpha, d, 2) \geq 2(1+s)-2 s \geq 0$. If $s=d-1$, then $\varphi(\alpha, d, 2)=$ $d-u \geq 0$ if and only if $u \leq d$, or $\alpha^{2}<(d-1)^{2}+(d+1)=d^{2}-d+2$. The final assertion is (2.14).

Proof of Theorem A(c). Suppose $\alpha \leq d-1<\left(d^{2}-d+2\right)^{1 / 2}, \alpha \notin I_{d}$ and $(\alpha, d, k)$ is the bad triple specified by Lemma 2.10. Then $k \geq 3$ by Lemma 3.1 , but by $(2.12)$,

$$
\alpha^{k-1}-1<\alpha_{k-1} \leq(\alpha-1) /(d-\alpha) \leq \alpha-1
$$

since $d-\alpha \geq 1$, which contradicts $\alpha \geq 1$.

In the remaining seven cases, $\alpha \in[d-1, d)$; the proof of $\mathrm{A}(\mathrm{k})$ was started in the introduction.

Proof of Theorem A(k). By Lemma 3.1, $\varphi(\alpha, d, 2)<0$ if $\left(d^{2}-d+2\right)^{1 / 2} \leq$ $\alpha<d ;(\alpha, d, 2)$ is bad, so by Lemmas 2.7 and 2.10, $\alpha \notin I_{d}$ and we have $\left[\left[d^{2}-d+1, \alpha_{2}-1\right]\right] \subseteq T_{d}(\alpha)$. 
Proofs of Theorems A(e), A(f) and A(h). These proofs are identical in form. We give enough of the vector $\underline{\alpha}$ to show that $\varphi(\alpha, d, k) \leq-1$ for appropriate $k$. If $13^{1 / 4} \leq \alpha<7^{1 / 3}$, then $\underline{\alpha}=(1,1,3,6,13, \ldots)$ and $\varphi(\alpha, 2,4)=1+1+1+3+$ $6-13=-1$, so $\alpha_{4}-1=12 \in T_{2}(\alpha)$. If $7^{1 / 3} \leq \alpha<2$, then $\underline{\alpha}=(1,1,3,7, \ldots)$ and $\varphi(\alpha, 2,3)=1+1+1+3-7=-1$, so $\alpha_{3}-1=6 \in T_{2}(\alpha)$. If $22^{1 / 3} \leq \alpha<$ $8^{1 / 2}$, then $\underline{\alpha}=(1,2,7,22, \ldots)$ and $\varphi(\alpha, 3,3)=1+2(1+2+7)-22=-1$, so $\alpha_{3}-1=21 \in T_{3}(\alpha)$.

In the final three cases, $d-1 \leq \alpha<\left(d^{2}-d+2\right)^{1 / 2}$; we show that $\alpha \in I_{d}$ by deriving a contradiction from Lemmas 2.10 and 3.1.

Proof of Theorem $\mathrm{A}(\mathrm{j})$. Suppose $(\alpha, d, k)$ is bad, then $k \geq 3$ by Lemma 3.1. By (2.13),

$$
1>(d-\alpha)\left(1+\alpha+\cdots+\alpha^{k-2}\right) \geq(d-\alpha)(1+\alpha) .
$$

Thus, $H(\alpha)>0$, where

$$
H(x)=1-(d-x)(1+x)=x^{2}-(d-1) x-(d-1) .
$$

Since $H$ is increasing for $x \geq(d-1) / 2$ and $\alpha<\left(d^{2}-d+2\right)^{1 / 2}$,

$$
0<H\left(\left(d^{2}-d+2\right)^{1 / 2}\right)=d^{2}-2 d+3-(d-1)\left(d^{2}-d+2\right)^{1 / 2} \text {. }
$$

A routine calculation shows that (3.6) is equivalent to the cubic inequality

$$
d^{3}-5 d^{2}+7 d-7<0
$$

which is false for $d=4$ by calculation, and for $d \geq 5$ by inspection.

The positive root of $H$ is $\beta=\beta(d)$, as defined in the statement of Theorem E. We shall need the observation that $\beta<\left(d^{2}-d+2\right)^{1 / 2}$ for $d \geq 4$.

Proof of Theorem A(g). We proceed as in the last case, except that we need to "bootstrap". Suppose $d=3,2<\alpha<22^{1 / 3}, \alpha \notin I_{3}$ and $(\alpha, 3, k)$ is bad. Again $k \geq 3$ by Lemma 3.1, and by (2.13),

$$
1>(3-\alpha)\left(1+\alpha+\cdots+\alpha^{k-2}\right) \geq(3-\alpha)(1+\alpha) \text {. }
$$

This gives a contradiction if $1 \leq \alpha \leq \mu=1+3^{1 / 2} \approx 2.73205$. If $\mu \leq \alpha<22^{1 / 3}$, then $\underline{\alpha}=(1,2,7,20$ or $21, \ldots)$, so $\varphi(\alpha, 3,3) \geq 1+2(1+2+7)-21=0$ and $(\alpha, 3,3)$ is not bad. Thus $k \geq 4$; as in (3.8),

$$
1>(3-\alpha)\left(1+\alpha+\alpha^{2}\right)>\left(3-22^{1 / 3}\right)\left(1+\mu+\mu^{2}\right) \approx 2.21640,
$$

which is a contradiction.

The final case is $A(d)$; its proof, like those of $A(e)$ and $A(f)$, may be deduced from [7]. We have included these proofs for completeness.

Proof of Theorem A(d). Suppose $\alpha<13^{1 / 4}, \alpha \notin I_{2}$ and $(\alpha, 2, k)$ is bad. By Lemma 3.1, $k \geq 3$; as in (3.8), (2.13) implies that

$$
(2-\alpha)(1+\alpha)<1 \text {. }
$$


so $\alpha>\frac{1}{2}\left(1+5^{1 / 2}\right)=\Phi \approx 1.61803$. We break the interval $\left[\Phi, 13^{1 / 4}\right)$ at $3^{1 / 2} \approx$ 1.73205. If $\alpha \in\left[\Phi, 3^{1 / 2}\right)$, then $\underline{\alpha}=(1,1,2,4$ or $5, \ldots)$; if $\alpha \in\left[3^{1 / 2}, 13^{1 / 4}\right)$, then $\underline{\alpha}=(1,1,3,5$ or $6, \ldots)$. In either case, $\varphi(\alpha, 2,3) \geq 0$ so $k \geq 4$. But now,

$$
(2-\alpha)\left(1+\alpha+\alpha^{2}\right)<1 .
$$

Solving the cubic, we find that $\alpha \geq 1.83929$. Thus $\underline{\alpha}=(1,1,3,6,11$ or $12, \ldots)$ and $\varphi(\alpha, 2,4) \geq 0$. Hence $k \geq 5$, and a final application of $(2.13)$ gives

$$
(2-\alpha)\left(1+\alpha+\alpha^{2}+\alpha^{3}\right)<1
$$

which implies $\alpha \geq 1.92746 \ldots \geq 13^{1 / 4}$, a contradiction at last.

This final argument does not contradict $\gamma=13^{1 / 4} \notin I_{2}$. Even though $\varphi(\gamma, 2,5)=1$, we have seen in the proof of $\mathrm{A}(\mathrm{e})$ that $\varphi(\gamma, 2,4)=-1$.

\section{THE PROOF OF THEOREM B}

In the notation of Theorem $\mathrm{A}$, there are five cases in which $\alpha \notin I_{d}$ : (a), (e), (f), (h) and (k). Theorem B states that $\left|T_{d}(\alpha)\right|=\infty$ in each of these cases. If $\alpha>d$, then $\left|T_{d}(\alpha)\right| \geq \alpha^{n}-d^{n}-1$ for all $n$ by the proof of Theorem $\mathrm{A}(\mathrm{a})$; this proves Theorem $\mathrm{B}(\mathrm{a})$. The remaining four cases are proved using a generic construction based on ideas from [7]. Given $m \in T_{d}(\alpha)$ and $m<\alpha_{n}$, let $m^{\prime}=m+(d-1) \alpha_{n}$. If we are fortunate, the equation $m^{\prime}=\sum \varepsilon_{i} \alpha_{i}$, $\varepsilon_{i} \in[[0, d-1]]$ implies $\varepsilon_{n}=d-1$, a contradiction, so that $m^{\prime} \in T_{d}(\alpha)$ as well. If we are especially fortunate, we may use $m^{\prime}$ as the new $m$, and pick a new $n^{\prime}$ to repeat the argument. This gives an infinite increasing sequence in $T_{d}(\alpha)$. We need two lemmas to implement this construction.

Lemma 4.1. If $m \in T_{d}(\alpha)$ and $m$ satisfies the inequalities

$$
\alpha_{n+1}-(d-1) \alpha_{n}>m>(d-1) \sum_{i=0}^{n-1} \alpha_{i}-\alpha_{n},
$$

then $m^{\prime}=m+(d-1) \alpha_{n} \in T_{d}(\alpha)$.

Proof. Following the argument outlined above, suppose, to the contrary, that $m^{\prime}=\sum_{i=0}^{\infty} \varepsilon_{i} \alpha_{i} \in S_{d}(\alpha)$. Since $\alpha_{n+1}>m^{\prime}$ by (4.2), $\varepsilon_{i}=0$ for $i \geq n+1$. If $\varepsilon_{n}<d-1$, then

$$
m^{\prime} \leq(d-2) \alpha_{n}+(d-1) \sum_{i=0}^{n-1} \alpha_{i},
$$

which violates the other inequality of (4.2). Thus $\varepsilon_{n}=d-1$, so that $m=$ $m^{\prime}-\varepsilon_{n} \alpha_{n}=\sum_{i=0}^{n-1} \varepsilon_{i} \alpha_{i} \in S_{d}(\alpha)$, a contradiction. 
Lemma 4.4. Suppose $b \in T_{d}(\alpha)$ and, for some $r \geq 1$, the following two inequalities hold:

$$
\begin{gathered}
\alpha_{r+1}-(d-1) \alpha_{r}>b>(d-1) \sum_{i=0}^{r-1} \alpha_{i}-\alpha_{r}, \\
\alpha^{r}\left(\alpha^{2}-(d-1) \alpha-1\right)>1 .
\end{gathered}
$$

Define the sequence $\left(b_{k}\right)$ by $b_{0}=b$ and

$$
b_{k}=b+(d-1) \sum_{i=0}^{k-1} \alpha_{r+2 i}, \quad k \geq 1 .
$$

Then $b_{k} \in T_{d}(\alpha)$ for all $k \geq 0$.

Proof. We apply Lemma 4.1 with $m=b_{k}, m^{\prime}=b_{k+1}$ and $n=r+2 k$; let

$$
\begin{aligned}
& E(k)=\alpha_{r+2 k+1}-(d-1) \alpha_{r+2 k}-b_{k}, \\
& F(k)=b_{k}-(d-1) \sum_{i=0}^{r+2 k-1} \alpha_{i}+\alpha_{r+2 k} .
\end{aligned}
$$

We want to show that $E(k)>0$ and $F(k)>0$ for all $k \geq 0$. For $k=0$, this is (4.5). Observe that

$$
\begin{aligned}
& E(k+1)-E(k)=\alpha_{r+2 k+3}-(d-1) \alpha_{r+2 k+2}-\alpha_{r+2 k+1}, \\
& F(k+1)-F(k)=\alpha_{r+2 k+2}-(d-1) \alpha_{r+2 k+1}-\alpha_{r+2 k},
\end{aligned}
$$

so we are done if we can show that

$$
D(s)=\alpha_{s+2}-(d-1) \alpha_{s+1}-\alpha_{s} \geq 0
$$

for $s \geq r$. Using $\alpha^{m} \geq \alpha_{m}>\alpha^{m}-1$ and (4.6), we have

$$
\begin{aligned}
D(s) & =\alpha_{s+2}-(d-1) \alpha_{s+1}-\alpha_{s} \geq \alpha^{s+2}-1-(d-1) \alpha^{s+1}-\alpha^{s} \\
& =\alpha^{s-r} \alpha^{r}\left(\alpha^{2}-(d-1) \alpha-1\right)-1>\alpha^{s-r}-1 \geq 0,
\end{aligned}
$$

which establishes (4.12), and completes the proof.

The first three cases are proved by a direct application of Lemma 4.4; the fourth, involving the parameter $d$, is more complicated.

Proofs of Theorems $\mathrm{B}(\mathrm{e}), \mathrm{B}(\mathrm{f})$, and $\mathrm{B}(\mathrm{h})$. As with Theorem A, the proofs in these three cases are virtually identical. In each case we use the element of $T_{d}(\alpha)$ cited in Theorem A. We only prove case (e) in detail. Suppose $d=2$ and $\alpha \in\left[13^{1 / 4}, 7^{1 / 3}\right)$, and let $b=12$ and $r=5$ as in Lemma 4.4. Then $\underline{\alpha}=\left(1,1,3,6,13, \alpha_{5}, \alpha_{6}, \ldots\right)$, where $\alpha_{5}=24$ or 25 and $\alpha_{6} \geq 46$. Then (4.5) becomes

$$
\alpha_{6}-\alpha_{5}>12>24-\alpha_{5}
$$


which is clearly true. For (4.6), note that $\alpha^{5}\left(\alpha^{2}-\alpha-1\right)$ is a product of increasing functions on $[1, \infty)$, and its value at $\alpha=13^{1 / 4}$ is $17.445 \ldots>1$. It follows from Lemma 4.4 that the sequence

$$
b_{0}=12, \quad b_{k}=12+\sum_{i=0}^{k-1} \alpha_{5+2 i}, \quad k \geq 1,
$$

lies in $T_{2}(\alpha)$.

For case (f), take $d=2, \alpha \in\left[7^{1 / 3}, 2\right), b=6$ and $r=4$. Then $\underline{\alpha}=$ $\left(1,1,3,7, \alpha_{4}, \alpha_{5}, \ldots\right)$ with $13 \leq \alpha_{4} \leq 15$ and $\alpha_{5} \geq 25$. The same reasoning shows that $6+\sum_{i=0}^{k-1} \alpha_{4+2 i} \in T_{2}(\alpha)$. For case $(\mathrm{h})$, take $d=3, \alpha \in\left[22^{1 / 3}, 8^{1 / 2}\right)$ and $b=21$ and $r=4$. Then $\underline{\alpha}=\left(1,2,7,22, \alpha_{4}, \alpha_{5}, \ldots\right)$ with $61 \leq \alpha_{4} \leq 63$ and $\alpha_{5} \geq 172$, and $21+2 \sum_{i=0}^{k-1} \alpha_{4+2 i} \in T_{3}(\alpha)$.

Proof of Theorem $\mathrm{B}(\mathrm{k})$. In this case, $\alpha \in\left[\left(d^{2}-d+2\right)^{1 / 2}, d\right)$ with $d \geq 3$; choose $b=d^{2}-d+1$ and $r=3$. Then (4.5) and (4.6) are:

$$
\begin{gathered}
\alpha_{4}-(d-1) \alpha_{3}>d^{2}-d+1>(d-1)\left(\alpha_{0}+\alpha_{1}+\alpha_{2}\right)-\alpha_{3}, \\
\alpha^{3}\left(\alpha^{2}-(d-1) \alpha-1\right)>1 .
\end{gathered}
$$

Using $\alpha_{0}=0, \alpha_{1}=d-1, \alpha_{i}>\alpha^{i}-1$ and $\alpha \geq \max \left(d-\frac{1}{2}, 8^{1 / 2}\right)$, we have:

$$
\begin{gathered}
\alpha_{4}-(d-1) \alpha_{3}-\left(d^{2}-d+1\right)>\alpha^{4}-(d-1) \alpha^{3}-\left(d^{2}-d+2\right) \\
\geq \alpha^{2}\left(\alpha^{2}-(d-1) \alpha-1\right) \geq \alpha^{2}\left(\alpha \cdot \frac{1}{2}-1\right)>0, \\
d^{2}-d+1-(d-1)\left(d+\alpha_{2}\right)+\alpha_{3} \geq 1-(d-1) \alpha^{2}+\alpha^{3}-1 \\
=\alpha^{2}(\alpha-(d-1)) \geq 0, \\
\alpha^{3}\left(\alpha^{2}-(d-1) \alpha-1\right) \geq \alpha^{3}\left(\alpha \cdot \frac{1}{2}-1\right) \geq 8^{3 / 2}\left(2^{1 / 2}-1\right)>1 .
\end{gathered}
$$

Thus, $d^{2}-d+1+(d-1) \sum_{i=0}^{k-1} \alpha_{3+2 i} \in T_{d}(\alpha)$.

\section{The GREedY REPRESENTATION AND THE PROOFS OF THEOREMS C AND D}

In this section we try to represent integers in the form $\sum \varepsilon_{i} \alpha_{i}, \varepsilon_{i} \in[[0, d-1]]$ when $\alpha<d$ using the greedy algorithm. If $\alpha \notin I_{d}$, this cannot be done for infinitely many integers by Theorem B. Nevertheless, a truncated version of the greedy algorithm is possible, and leads to the proofs of Theorems C and D. (The index $t=t(\alpha, d)$ below is called the cutoff value for $(\alpha, d)$.)

Lemma 5.1 (The greedy representation). Suppose $\alpha<d$ and $t$ is the smallest integer so that $(d-\alpha) \alpha_{t} \geq \alpha-1$. Then every integer $m$ can be written:

$$
m=\sum_{i=t}^{\infty} \varepsilon_{i}(m) \alpha_{i}+u(m), \quad \varepsilon_{i}(m) \in[[0, d-1]], u(m) \in\left[\left[0, \alpha_{t}-1\right]\right]
$$


and, for all $m \geq 0$,

$$
u(m+1)=0 \text { or } u(m+1)=u(m)+1 .
$$

Proof. By Lemma 2.11,s $\geq t$ and $\alpha_{s} \geq \alpha_{t} \geq(\alpha-1) /(d-\alpha)$ imply that $d \alpha_{s}-$ $\alpha_{s+1}>-1$, so $d \alpha_{s} \geq \alpha_{s+1}$. Let $I_{0}=\left[\left[0, \alpha_{t}-1\right]\right]$ and $I_{s}=\left[\left[\alpha_{s}, \alpha_{s+1}-1\right]\right]$ for $s \geq t$. We define $(\underline{\varepsilon}(m), u(m))$ first for $m \in I_{0}$ and $I_{t}$, and then, recursively, for $m \in I_{s}$ when $s>t$.

For $m \in I_{0}$, let $(\underline{\varepsilon}(m), u(m))=(\underline{0}, m)$; this satisfies (5.2). For $m \in I_{t}$, let $\varepsilon_{i}(m)=0$ for $i \geq t+1, \varepsilon_{t}(m)=\left[m / \alpha_{t}\right]$ and $u(m)=m-\varepsilon_{t}(m) \alpha_{t}$. Then $u(m) \in I_{0}$ and

$$
0 \leq \varepsilon_{t}(m) \leq\left[\left(\alpha_{t+1}-1\right) / \alpha_{t}\right] \leq\left[\left(d \alpha_{t}-1\right) / \alpha_{t}\right]<d,
$$

so (5.2) is verified. Suppose that every integer less than $\alpha_{s}-1$ has been written in the form (5.2) with $\varepsilon_{i}(m)=0$ for $i \geq s$. For $m \in I_{s}$, let $R(m)=m-$ $\left[m / \alpha_{s}\right] \alpha_{s}$, so $R(m) \leq \alpha_{s}-1$ and $\varepsilon_{i}(R(m))=0$ for $i \geq s$. Define $\underline{\varepsilon}(m)$ as follows: $\varepsilon_{i}(m)=0$ for $i \geq s+1, \varepsilon_{s}(m)=\left[m / \alpha_{s}\right](\leq d-1), \varepsilon_{i}(m)=\varepsilon_{i}(R(m))$ for $i \leq s-1$ and $u(m)=u(R(m))$. This verifies (5.2) for $m \in I_{s}$.

We must still check (5.3). Since $u\left(\alpha_{t}\right)=0$, (5.3) holds for $m \leq \alpha_{t}-1$. Suppose it holds for $m \leq \alpha_{s}-1$, and $m \in I_{s}$. If $m=\alpha_{s+1}-1$, then $m+1=$ $\alpha_{s+1}$ and $u(m+1)=0$; otherwise, $m, m+1 \in I_{s}$. If $\varepsilon_{s}(m)=\varepsilon_{s}(m+1)$, then $R(m+1)=R(m)+1$, so (5.3) holds by induction. If $\varepsilon_{s}(m)<\varepsilon_{s}(m+1)$, then $\alpha_{s}$ divides $m+1$, so $R(m+1)=0$ and $u(m+1)=0$.

Proof of Theorem C. Since $\alpha<d, \alpha \in I_{d+1}$ by Theorem A(c); thus $u(m)=$ $\sum \eta_{i} \alpha_{i} \in S_{d+1}(\alpha)$ with $\eta_{i} \in[[0, d]]$. As $u(m) \leq \alpha_{t}-1, \eta_{i}=0$ for $i \geq t$. After replacing $u(m)$ by $\sum \eta_{i} \alpha_{i}$ in (5.2), we obtain the desired representation.

Theorem B states that $\left|T_{d}(\alpha)\right|=\infty$ if $\alpha<d$ and $\alpha \in I_{d}$, but the set $\left(b_{k}\right)$ constructed in the proof is quite sparse in $\mathbf{Z}^{+}$. The following notation is standard. For $A \subseteq \mathbf{Z}^{+}$, let $N(A ; n)$ denote the number of integers $\leq n$ which belong to $A$. We define the lower density of $A, D(A)$ :

$$
D(A)=\liminf _{n \rightarrow \infty} \frac{N(A ; n)}{n+1} .
$$

Theorem $\mathrm{D}$ asserts that $D\left(S_{d}(A)\right)>0$ when $\alpha<d$.

Proof of Theorem D. Fix $\alpha<d$ with $\alpha \notin I_{d}$, and define $t$ and $u(m)$ as above. Since $u(m) \leq \alpha_{t}-1$, any representation $u(m)=\sum \varepsilon_{i} \alpha_{i}$ has $\varepsilon_{i}=0$ for $i \geq t$. Thus, if $u(m) \in S_{d}(\alpha)$, then (5.2) shows that $m \in S_{d}(\alpha)$. It therefore suffices to show that $\left\{m: u(m) \in S_{d}(\alpha)\right\}$ has positive lower density. By (5.3), every interval $[[0, n]]$ can be written as a disjoint union of blocks $W_{i}=\left[\left[w_{i}, w_{i+1}-1\right]\right]$ so that $u(j)=j-w_{i}$ for $j \in W_{i}$. Let

$$
\lambda=\lambda(\alpha, d)=\min \left\{(k+1)^{-1} N\left(S_{d}(\alpha), k\right): k \in\left[\left[0, \alpha_{t}-1\right]\right]\right\} ;
$$

since $[[0, d-1]] \subseteq S_{d}(\alpha), \lambda>0$. As $W_{i}$ contains $\geq \lambda\left(w_{i+1}-w_{i}\right)$ elements of $S_{d}(\alpha), N\left(S_{d}(\alpha), n\right) \geq \lambda(n+1)$ for all $n$, so $D\left(S_{d}(\alpha)\right) \geq \lambda>0$. 
For any fixed $(\alpha, d), \lambda(\alpha, d)$ can be readily computed. For example, if $\alpha \in$ $\left[13^{1 / 4}, 7^{1 / 3}\right.$ ) and $d=2$, then $t=4$ and $\lambda=12 / 13$. Since $t \rightarrow \infty$ as $\alpha \rightarrow d$, the computations become extremely unpleasant. If Conjecture $F$ is true, then the question is moot.

This greedy representation of $m$ may not be the only one satisfying (5:2). Fix $(\alpha, d)$ with $\alpha<d$, and for $r \geq t(\alpha, d)$, let $E_{r}=E_{r}(\alpha, d)$ denote the set of strings $(\underline{\varepsilon}, u)=\left(\varepsilon_{r}, \ldots, \varepsilon_{t}, u\right)$ with $\varepsilon_{i} \in[[0, d-1]]$ and $u \in\left[\left[0, \alpha_{t}-1\right]\right]$. Let

$$
f(\underline{\varepsilon}, u)=\sum_{i=t}^{r} \varepsilon_{i} \alpha_{i}+u
$$

Then the greedy representation of $m$ gives $m=f(\underline{\varepsilon}(m), u(m))$. Since there are $\alpha_{t} d^{r-t+1}$ strings in $E_{r}$ and $0 \leq f(\underline{\varepsilon}, u) \leq \alpha_{t}-1+(d-1) \sum_{i=t}^{r} \alpha_{i}<c_{0} \alpha^{r}$, an integer in $\left[\left[0, \alpha^{r}\right]\right]$ has $\approx c_{1}(d / \alpha)^{r}$ representations on average. The greedy representations within $E_{r}$ can be characterized as the maximal elements of the level sets $\left\{f(\underline{\varepsilon}, u)=m:(\underline{\varepsilon}, u) \in E_{r}\right\}$ under the lexicographic ordering.

Lemma 5.8. Suppose $m=f(\underline{\varepsilon}(m), u(m))=f(\underline{\eta}, v)$, where $(\underline{\eta}, v) \in E_{r}$, and suppose $\eta_{i}=\varepsilon_{i}(m)$ for $i \geq k+1$. Then $\varepsilon_{k}(m) \geq \eta_{k}$.

Proof. First suppose $k<r$. We have

$$
m=\sum_{i=t}^{r} \varepsilon_{i}(m) \alpha_{i}+u(m)=\sum_{i=t}^{r} \eta_{i} \alpha_{i}+v .
$$

As usual, let $R(m)=m-\varepsilon_{r}(m) \alpha_{r} ; \varepsilon_{i}(m)=\varepsilon_{i}(R(m))$ for $i \leq r-1$. Thus, we may cancel $\varepsilon_{r}(m) \alpha_{r}=\eta_{r} \alpha_{r}$ from both sides of (5.9) while preserving the fact that the first representation is greedy. This process may be repeated so that we assume that $m \leq \alpha_{k+1}-1$, and

$$
m=\sum_{i=t}^{k} \varepsilon_{i}(m) \alpha_{i}+u(m)=\sum_{i=t}^{k} \eta_{i} \alpha_{i}+v .
$$

But $\eta_{k} \leq m / \alpha_{k}$ and $\varepsilon_{k}(m)=\left[m / \alpha_{k}\right]$, so $\varepsilon_{k}(m) \geq \eta_{k}$.

\section{THE PROOF OF THEOREM E}

A PV (or Pisot-Vijayoragnavan) number is an algebraic integer $\alpha=\alpha_{1}$ whose conjugates, $\alpha_{2}, \ldots, \alpha_{m}$, all lie in $|z|<1$. These numbers have been extensively studied for their algebraic and analytic properties (see $\S \S \mathrm{K} 25$ and R06 in [9] and [14].) Suppose $\alpha$ is a PV number with minimal polynomial $p(x)=x^{m}+\sum b_{i} x^{i} \in \mathbf{Z}[x]$, and let $a_{n}=\sum \alpha_{j}^{n}$. By the elementary theory of symmetric polynomials, $a_{n}$ is integral for $n \geq 0$; moreover, $\left(a_{n}\right)$ satisfies the linear recurrence called Newton's identity:

$$
a_{n+m}+\sum_{i=0}^{m-1} b_{i} a_{n+i}=\sum_{j=1}^{m} \alpha_{j}^{n} p\left(\alpha_{j}\right)=0 .
$$


Since $\alpha$ is PV, $\alpha_{j}^{n} \rightarrow 0$ for $j \geq 2$, and so $a_{n} \approx \alpha^{n}$. If $m=2$ and $\alpha_{2} \in(-1,0)$, then $a_{n}=\left[\alpha^{n}\right]$ or $\left[\alpha^{n}\right]+1$, depending on the parity of $n \bmod 2$. Using (6.1), we can always obtain an explicit linear recurrence for $\left[\alpha^{n}\right]=\alpha_{n}$.

We are interested in one particular PV number. Fix $d \geq 4$ and let

$$
\beta=\beta(d)=\frac{1}{2}\left(d-1+\left(d^{2}+2 d-3\right)^{1 / 2}\right) .
$$

As previously noted, $\beta$ is the positive root of $H(x)=x^{2}-(d-1) x-(d-1)$ (cf. (3.5)) and, as $d>\beta \geq\left(d^{2}-d+2\right)^{1 / 2}, \beta \notin I_{d}$. (It turns out that $\beta(d) \in I_{d}$ for $d \leq 3$.) Since the conjugate of $\beta$ lies in $(-1,0), \beta$ is a PV number. We show in Lemma 6.7 that $\beta_{n}=\left[\beta^{n}\right]$ satisfies the second-order recurrence:

$$
\beta_{n+2}=(d-1)\left(\beta_{n+1}+\beta_{n}\right)+\delta_{n} \text {, }
$$

where

$$
\delta_{n}=d-\frac{3}{2}-\frac{1}{2}(-1)^{n} .
$$

By Lemma 5.1, every integer $m$ can be written greedily as (5.2), where $\varepsilon_{i}(m) \in[[0, d-1]]$ and $u(m) \in\left[\left[0, \beta_{t}-1\right]\right]$. It turns out that $t=t(d, \beta)$ always equals 2 (Lemma 6.10) and $\beta_{2}=d^{2}-2$. We shall write $\left[\left[0, \beta_{2}-1\right]\right]=J_{1} \cup J_{2}$, where $J_{1}=\left[\left[0, d^{2}-d\right]\right]$ and $J_{2}=\left[\left[d^{2}-d+1, d^{2}-3\right]\right]$. As $(d-1)\left(\beta_{0}+\beta_{1}\right)=$ $d^{2}-d$, if $u(m) \in J_{1}$, then $u(m)=\varepsilon_{0} \beta_{0}+\varepsilon_{1} \beta_{1}$ for $\varepsilon_{i}=[[0, d-1]]$ and $m \in S_{d}(\beta)$. If $u(m) \in J_{2}$, then $m$ may still belong to $S_{d}(\beta)$, provided there is a nongreedy way to write $m$ in the form $\sum \eta_{k} \beta_{k}$.

Using (6.3), we give a precise description of the strings $(\underline{\varepsilon}(m), u(m))$ which arise from greedy representations (Lemma 6.14). We also describe the alternative representations (Lemma 6.23). Suppose $u(m) \in J_{2}$ and $\varepsilon_{j+2}(m) \geq 1$ and $\varepsilon_{j+1}(m)=\varepsilon_{j}(m)=0$ with $j \geq 3$. By (6.3) we can "trade" one $\beta_{j+2}$ from the greedy representation for $d-1 \quad \beta_{j+1}$ 's and $d-1 \quad \beta_{j}$ 's, with $\delta_{j}$ left over, which we add to $u(m)$ to obtain one more $\beta_{2}$ (if $u(m) \in J_{2}$, then $\varepsilon_{2}(m) \leq d-2$ ) and a smaller $u^{\prime}(m)$ which now lies in $J_{1}$. It turns out that, if the pattern $(\geq 1,0,0, \geq 1)$ does not occur in $\underline{\varepsilon}(m)$, then $m \in T_{d}(\beta)$. This reduces to a purely combinatorial counting problem; we show that

$$
N_{r}=N\left(T_{d}(\beta) ; \beta_{r+1}-1\right)=c(d) \sigma^{r}+O(1),
$$

where

$$
\sigma=\sigma(d)=\frac{1}{2}\left(d-1+\left(d^{2}+2 d-7\right)^{1 / 2}\right) .
$$

Since $\beta_{r+1} / \beta_{r} \rightarrow \beta$, it follows that $c_{1} n^{e(d)} \leq N\left(T_{d}(\beta) ; n\right) \leq c_{2} n^{e(d)}$ for some $c_{i}>0$, where $e(d)=\log _{\beta(d)} \sigma(d)<1$. Since $e(d)<1, N\left(T_{d}(\beta) ; n\right)=o(n)$ and $S_{d}(\beta)$ has density 1 , which proves Theorem E.

We begin with two lemmas which give the recurrence for $\beta_{n}$ and, with Lemma 5.1, establish that $t(\beta(d), d)=2$ for all $d \geq 4$. 
Lemma 6.7. $\left(\beta_{n}\right)$ satisfies (6.3).

Proof. The minimal polynomial of $\beta$ is $H(x)=x^{2}-(d-1) x-(d-1)$ and $\bar{\beta}=-(d-1) / \beta \in(-1,0)$; let $b_{n}=\beta^{n}+\bar{\beta}^{n}$ for $n \geq 0$. By $(6.1)$,

$$
b_{n+2}-(d-1)\left(b_{n+1}+b_{n}\right)=0 \text {; }
$$

since $b_{0}=2$ and $b_{1}=d-1, b_{n} \in \mathbf{Z}$. Since $\bar{\beta}^{n}$ lies alternately in $(-1,0)$ and $(0,1), \beta_{n}=\left[\beta^{n}\right]=b_{n}-\frac{1}{2}\left(1+(-1)^{n}\right)$, and (6.3) follows from (6.8).

For later use, we record the first few values of $\beta_{i}$ :

$$
\beta_{0}=1, \quad \beta_{1}=d-1, \quad \beta_{2}=d^{2}-2, \quad \beta_{3}=d^{3}-3 d+2 .
$$

\section{Lemma 6.10.}

$$
\beta_{2} \geq(\beta-1) /(d-\beta)>\beta_{1} .
$$

Proof. A calculation shows that

$$
(\beta-1) /(d-\beta)=\frac{1}{2}\left\{\left(d^{2}-3\right)+(d-1)\left(d^{2}+2 d-3\right)^{1 / 2}\right\} \text {. }
$$

The first inequality in (6.11) follows from putting $d^{2}+2 d-3<(d+1)^{2}$ in (6.12), and (6.9); the second is immediate.

We now analyze greedy strings. Recall that $E_{r}$ is the set of strings $(\underline{\varepsilon}, u)=$ $\left(\varepsilon_{r}, \ldots, \varepsilon_{2}, u\right)$ with $\varepsilon_{i} \in[[0, d-1]]$ and $u \in J_{1} \cup J_{2}=\left[\left[0, d^{2}-3\right]\right]$, and that $f(\underline{\varepsilon}, u)=\sum \varepsilon_{k} \beta_{k}+u$. Let

$$
\widetilde{E}_{r}=\left\{(\underline{\varepsilon}, u) \in E_{r}:(\underline{\varepsilon}, u)=(\underline{\varepsilon}(m), u(m)) \text { for some } m<\beta_{r+1}\right\}
$$

denote the set of strings which arise from greedy representations. We shall say that $(i, j)$ occurs at $k$ in $(\underline{\varepsilon}, u)$ if $\varepsilon_{k+1}=i$ and $\varepsilon_{k}=j$ and that $(i, j)$ does not occur in $(\underline{\varepsilon}, u)$ if it occurs at no $k$.

Lemma 6.14. For $r \geq 2, \widetilde{E}_{r}$ consists of all strings $(\underline{\varepsilon}, u)$ in $E_{r}$ satisfying one of the following three disjoint conditions $\left(\delta_{j}\right.$ has its previous meaning):

(6.15)(i) $\varepsilon_{2}<d-1$ and $(d-1, d-1)$ does not occur,

(6.15)(ii) $\varepsilon_{2}=d-1, u \in\left[\left[0, d^{2}-d-1\right]\right]$ and $(d-1, d-1)$ does not occur,

(6.15)(iii) $(d-1, d-1)$ does not occur at $k>j,(d-1, d-1)$ occurs at $j$ and $\left(\varepsilon_{j-1}, \ldots, \varepsilon_{2}, u\right)=(0, \ldots, 0, u)$, where $u \in\left[\left[0, \delta_{j}-1\right]\right]$.

Proof. Let $\bar{E}_{r}$ denote the set of strings in $E_{r}$ which satisfy one of the conditions in (6.15). We give a recursive description of $\bar{E}_{r}$, and then show that $\widetilde{E}_{r}$ satisfies the same recursion.

It is easy to check that

$$
\overline{E_{2}}=\left(\bigcup_{i}(i, u)\right) \cup\left(\bigcup_{v}(d-1, v)\right)
$$

$$
\overline{E_{3}}=\left(\bigcup_{i}\left(i, \bar{E}_{2}\right)\right) \cup\left(\bigcup_{i} \bigcup_{u}(d-1, i, u)\right) \cup\left(\bigcup_{w}(d-1, d-1, w)\right) ;
$$


where the unions are taken over the following: $i \in[[0, d-2]], u \in\left[\left[0, d^{2}-3\right]\right]$, $v \in\left[\left[0, d^{2}-d-1\right]\right]$ and $w \in\left[\left[0, \delta_{2}-1\right]\right]$. Fix $r \geq 4$. If $\varepsilon_{r} \leq d-2$, and $(\underline{\varepsilon}, u) \in E_{r-1}$, then $\left(\varepsilon_{r}, \underline{\varepsilon}, u\right) \in \bar{E}_{r}$ if and only if $(\underline{\varepsilon}, u) \in \bar{E}_{r-1}$. If $\varepsilon_{r}=d-1$, $\varepsilon_{r-1} \in[[0, d-2]]$ and $(\underline{\varepsilon}, u) \in E_{r-2}$, then $\left(\varepsilon_{r}, \varepsilon_{r-1}, \underline{\varepsilon}, u\right) \in \bar{E}_{r}$ if and only if $(\underline{\varepsilon}, u) \in \bar{E}_{r-2}$. Finally, if $\varepsilon_{r}=\varepsilon_{r-1}=d-1$, then $\left(\varepsilon_{r}, \varepsilon_{r-1}, \underline{\varepsilon}, v\right) \in \bar{E}_{r}$ if and only if $\underline{\varepsilon}=\underline{0}$ and $v \in\left[\left[0, \delta_{r-1}-1\right]\right]$. In other words,

$$
\begin{aligned}
\bar{E}_{r}= & \left(\bigcup_{i}\left(i, \bar{E}_{r-1}\right)\right) \cup\left(\bigcup_{i}\left(d-1, i, \bar{E}_{r-2}\right)\right) \\
& \cup\left(\bigcup_{z}(d-1, d-1,0, \ldots, 0, z)\right),
\end{aligned}
$$

where the unions in (6.18) are taken over $i \in[[0, d-2]]$ and $z \in\left[\left[0, \delta_{r-1}-1\right]\right]$.

Let $B_{s}(i, p)$ denote the block [ $\left.\left[i \beta_{s}, i \beta_{s}+p\right]\right]$, where $p \leq \beta_{s}-1$; if $m \in$ $B_{s}(i, p)$, then $\varepsilon_{s}(m)=i$ and $R(m)=p$. If $r=2$, then $(d-1, d-1)$ cannot occur. By (6.9), $\beta_{3}-1=d^{3}-3 d+1=(d-1) \beta_{2}+d^{2}-d-1$, so

$$
\left[\left[0, \beta_{3}-1\right]\right]=\left(\bigcup_{i=0}^{d-2} B_{2}\left(i, \beta_{2}-1\right)\right) \cup B_{2}\left(d-1, d^{2}-d-1\right) .
$$

If $m \in B_{2}\left(i, \beta_{2}-1\right)$, then $(\underline{\varepsilon}(m), u(m))=(i, R(m))$, where $i \in[[0, d-2]]$ and $R(m) \in\left[\left[0, \beta_{2}-1\right]\right]$. If $m \in B_{2}\left(d-1, d^{2}-d-1\right)$, then $(\underline{\varepsilon}(m), u(m))=$ $(d-1, R(m))$, where $R(m) \in\left[\left[0, d^{2}-d-1\right]\right]$. Comparing with (6.16), we see that $\widetilde{E}_{2}=\bar{E}_{2}$.

Similarly, $\beta_{4}-1=(d-1) \beta_{3}+(d-1) \beta_{2}+\delta_{2}-1$ by (6.3), so

$$
\left[\left[0, \beta_{4}-1\right]\right]=\left(\bigcup_{i=0}^{d-2} B_{3}\left(i, \beta_{3}-1\right)\right) \cup B_{3}\left(d-1,(d-1) \beta_{2}+\delta_{2}-1\right) .
$$

Since $\underline{\varepsilon}(m)=\left(\varepsilon_{s}(m), \underline{\varepsilon}(R(m))\right)$, with allowance for zeros at the left, the greedy representations for $m \in \bigcup_{i=0}^{d-2} B_{3}\left(i, \beta_{3}-1\right)$ simply amount to $\bigcup_{i=0}^{d-2}\left(i, \widetilde{E}_{2}\right)$. If $m \in B_{3}\left(d-1,(d-1) \beta_{2}+\delta_{2}-1\right)$, then $\varepsilon_{3}(m)=d-1$ and $R(m) \leq$ $(d-1) \beta_{2}+\delta_{2}-1$. Thus $(6.20)$ shows that

$$
\widetilde{E}_{3}=\left(\bigcup_{i}\left(i, \widetilde{E}_{2}\right)\right) \cup\left(\bigcup_{i} \bigcup_{u}\left(d-1, i, u_{1}\right)\right) \cup\left(\bigcup_{w}\left(d-1, d-1, u_{2}\right)\right) \text {, }
$$

where the unions are taken over $i \in[[0, d-2]], u \in\left[\left[0, \beta_{2}-1\right]\right]$, and $w \in$ [[0, $\left.\left.\delta_{2}-1\right]\right]$. That is, $\widetilde{E}_{3}=\bar{E}_{3}$.

Since $\beta_{r+1}-1=(d-1) \beta_{r}+(d-1) \beta_{r-1}+\delta_{r-1}-1$ by (6.3), the same reasoning applied to $\left[\left[0, \beta_{r+1}-1\right]\right]$ for $r \geq 4$ gives

$$
\begin{gathered}
E_{r}=\left(\bigcup_{i}\left(i, \widetilde{E}_{r-1}\right)\right) \cup\left(\bigcup_{i}\left(d-1, i, \widetilde{E}_{r-2}\right)\right) \\
\cup\left(\bigcup_{z}(d-1, d-1,0, \ldots, 0, z)\right),
\end{gathered}
$$


where the unions are taken over $i \in[[0, d-2]]$ and $z \in\left[\left[0, \delta_{r-1}-1\right]\right]$. This is the same recurrence, (6.18), satisfied by $\bar{E}_{r}$, so $\widetilde{E}_{r}=\bar{E}_{r}$ for all $r \geq 2$.

As noted earlier, if $u(m) \in J_{1}$, then the greedy representation shows directly that $m \in S_{d}(\beta)$. If $u(m) \in J_{2}$ and $m=\sum \eta_{k} \beta_{k} \in S_{d}(\beta)$, then Lemma 6.14 gives us enough information about $(\underline{\varepsilon}(m), u(m))$ to determine whether $m$ has an alternative representation.

Lemma 6.23. Suppose $m \in\left[\left[0, \beta_{r+1}-1\right]\right]$ and $u(m) \in J_{2}$. Then $m \in S_{d}(\beta)$ if and only if there exists $j \geq 3$ so that

$$
\varepsilon_{j}(m)=\varepsilon_{j+1}(m)=0 \text { and } \varepsilon_{j+2}(m)>0 .
$$

Proof. Since $u(m) \geq d^{2}-d$ for $m \in J_{2}$ and $\delta_{j} \leq d-1$, neither (6.15)(ii) nor (iii) holds for $(\underline{\varepsilon}(m), u(m))$, thus (6.15)(i) holds: $\varepsilon_{2}(m)<d-1$ and $(d-1, d-1)$ does not occur in $\underline{\varepsilon}(m)$. Suppose (6.24) holds for $j \geq 3$; we define $(\underline{\eta}, v)$ by:

$$
\begin{aligned}
& \eta_{j+2}=\varepsilon_{j+2}(m)-1, \quad \eta_{j+1}=\eta_{j}=d-1, \\
& \eta_{2}=\varepsilon_{2}(m)+1, \quad \eta_{i}=\varepsilon_{i}(m) \text { otherwise, and } \\
& v=u(m)-\left(d^{2}-2\right)+\delta_{j} .
\end{aligned}
$$

As $\delta_{j}=d-2$ or $d-1$ and $u(m) \in J_{2}, v \in[[1, d-2]] \subset J_{1}$. Since $\varepsilon_{2}(m) \leq d-2$ and $\varepsilon_{j+2}(m) \geq 1, \eta_{i} \in[[0, d-1]]$ for all $i$ and $(\underline{\eta}, v) \in E_{r}$. Finally,

$$
\begin{aligned}
f(\underline{\eta}, v) & =f(\underline{\varepsilon}, u)-\beta_{j+2}+(d-1)\left(\beta_{j+1}+\beta_{j}\right)+\beta_{2}+\delta_{j}-\left(d^{2}-2\right) \\
& =m-\delta_{j}+\beta_{2}+\delta_{j}-\beta_{2}=m,
\end{aligned}
$$

so $m \in S_{d}(\beta)$.

Suppose now that (6.24) holds for no $j \geq 3$ : there are no "internal" consecutive 0's in $\underline{\varepsilon}(m)$. We shall show that $m \in T_{d}(\beta)$. If $r=2$ and

$$
m=\varepsilon_{2}(m) \beta_{2}+u(m)=\eta_{2} \beta_{2}+\eta_{1} \beta_{1}+\eta_{0} \beta_{0} \in S_{d}(\beta),
$$

then $\eta_{1} \beta_{1}+\eta_{0} \beta_{0} \equiv u(m)\left(\bmod \beta_{2}\right)$. This is a contradiction, since $\eta_{1} \beta_{1}+\eta_{0} \beta_{0} \in$ $J_{1}$ and $u(m) \in J_{2}$. Suppose $r \geq 3$ and

$$
m=\sum_{i=2}^{r} \varepsilon_{i}(m) \beta_{i}+u(m)=\sum_{i=0}^{r} \eta_{i} \beta_{i}
$$

and suppose $\varepsilon_{i}(m)=\eta_{i}$ for $i \geq s+1$, but $\varepsilon_{s}(m) \neq \eta_{s}$. By Lemma 5.8, $\varepsilon_{s}(m)>\eta_{s}$; consider the truncated integer $n$ :

$$
n=\left(\varepsilon_{s}(m)-\eta_{s}\right) \beta_{s}+\sum_{i=2}^{s-1} \varepsilon_{i}(m) \beta_{i}+u(m)=\sum_{i=0}^{s-1} \eta_{i} \beta_{i} .
$$

(By the preceding argument, $s \geq 3$.) By hypothesis, at least one of each pair $\left\{\varepsilon_{s-2 k-1}, \varepsilon_{s-2 k-2}\right\}$ is positive for $s-2 k-2 \geq 3$. Since $\varepsilon_{s}(m)-\eta_{s} \geq 1$ and 
$u(m) \geq d^{2}-d+1$, we obtain from the first expression for $n$ the inequalities:

(6.30)(i) $\quad n \geq \beta_{s}+\beta_{s-2}+\cdots+\beta_{4}+d^{2}-d+1 \quad(s$ even, $s \geq 4)$

(6.30)(ii) $n \geq \beta_{s}+\beta_{s-2}+\cdots+\beta_{3}+d^{2}-d+1 \quad$ (s odd, $s \geq 3$ ).

A repeated application of $(6.3)$ to $(6.30)$ gives

$$
n \geq \sum_{i=2}^{s-1}(d-1) \beta_{i}+\left(\frac{s}{2}-1\right) \delta_{s}+d^{2}-d+1 \quad(s \text { even, } s \geq 4),
$$

$$
n \geq \sum_{i=1}^{s-1}(d-1) \beta_{i}+\frac{1}{2}(s-1) \delta_{s}+d^{2}-d+1 \quad(s \text { odd }, s \geq 3) .
$$

But, by the second expression for $n$ in (6.29),

$$
n=\sum_{i=0}^{s-1} \eta_{i} \beta_{i} \leq(d-1) \sum_{i=1}^{s-1} \beta_{i}+(d-1),
$$

which contradicts either inequality in (6.31). Thus (6.28) is impossible, so $m \in T_{d}(\beta)$. This completes the proof.

We may summarize our discussion as follows.

Lemma 6.33. Suppose $m \leq \beta_{r+1}$. Then $m \in T_{d}(\beta)$ if and only if $(\underline{\varepsilon}(m), u(m))$ $\in E_{r}^{*}$, where $E_{r}^{*}$ is the set of $(\underline{\varepsilon}, u)$ in $E_{r}$ for which $u \in J_{2}, \varepsilon_{2}<d-1$, and neither $(d-1, d-1)$ nor $(\geq 1,0,0, \geq 1)$ occurs.

Let $Y(r)$ denote the set of strings $\left(\varepsilon_{r}, \ldots, \varepsilon_{3}\right) \in[[0, d-1]]^{r-2}$ such that $(d-1, d-1)$ does not occur and $(0,0)$ does not occur, except initially, and let $F(r)=|Y(r)|$. Then $(\underline{\varepsilon}, u) \in E_{r}^{*}$ if and only if $\left(\varepsilon_{r}, \ldots, \varepsilon_{3}\right) \in Y(r)$, $\varepsilon_{2} \in[[0, d-2]]$ and $u \in J_{2}$. Since $\left|J_{2}\right|=d-3$, it follows that

$$
N_{r}=N\left(T_{d}(\beta) ; \beta_{r+1}-1\right)=(d-2)(d-3) F(r) .
$$

We compute $N_{r}$ from (6.34), by describing $Y(r)$ recursively.

Lemma 6.35. There are constants $c_{i}$ so that

$$
F(r)=c_{1} \sigma^{r}+c_{2} \bar{\sigma}^{r}+c_{3}+c_{4}(-1)^{r},
$$

where $\sigma(c f .(6.6))$ and $\bar{\sigma}=d-1-\sigma$ are the roots of the quadratic $\widetilde{H}(x)=$ $x^{2}-(d-1) x-(d-2)$.

Proof. We divide $Y(r)=\bigcup Y_{i}(r)$ into four subclasses. Let

$$
\begin{gathered}
Y_{1}(3)=\varnothing, \quad Y_{2}(3)=\{(0)\} \\
Y_{3}(3)=\{(1), \ldots,(d-2)\}, \quad Y_{4}(3)=\{(d-1)\}
\end{gathered}
$$


and, for $r \geq 4$, let

$$
\begin{aligned}
& Y_{1}(r)=\left\{\underline{\varepsilon} \in Y(r): \varepsilon_{r}=\varepsilon_{r-1}=0\right\}, \\
& Y_{2}(r)=\left\{\underline{\varepsilon} \in Y(r): \varepsilon_{r}=0, \varepsilon_{r-1} \neq 0\right\}, \\
& Y_{3}(r)=\left\{\underline{\varepsilon} \in Y(r): 1 \leq \varepsilon_{r} \leq d-2\right\}, \\
& Y_{4}(r)=\left\{\underline{\varepsilon} \in Y(r): \varepsilon_{r}=d-1\right\} .
\end{aligned}
$$

For $r \geq 3$, the strings in $Y(r+1)$ arise by prefixing any $\varepsilon_{r+1} \in[[0, d-1]]$ to any string in $Y(r)$, with two exceptions. If $\underline{\varepsilon} \in Y_{1}(r)$, then $\varepsilon_{r+1}=0$; if $\underline{\varepsilon} \in Y_{4}(r)$, then $\varepsilon_{r+1} \neq d-1$. Thus, $F_{i}(r)=\left|Y_{i}(r)\right|$ satisfies the following recurrence:

$$
F_{1}(r+1)=F_{1}(r)+
$$

$$
\begin{array}{rrr}
F_{2}(r)+ & F_{3}(r)+ & F_{4}(r) \\
(d-2) F_{2}(r)+(d-2) F_{3}(r) & +(d-2) F_{4}(r) \\
F_{2}(r)+ & F_{3}(r)
\end{array}
$$

(6.39)(ii) $\quad F_{2}(r+1)=$$$
\text { (6.39)(iii) } F_{3}(r+1)=
$$$$
\text { (6.39)(iv) } F_{4}(r+1)=
$$

Each $F_{i}$ (and so $F=\sum F_{i}$ ) satisfies the recurrence whose auxiliary equation is the characteristic polynomial of the matrix implicit in (6.39), namely $\left(\lambda^{2}-1\right)\left(\lambda^{2}-(d-1) \lambda-(d-2)\right)$. That is, for $r \geq 3$,

$$
F(r+4)=(d-1)\{F(r+3)+F(r+2)-F(r+1)\}-(d-2) F(r) .
$$

This can also be directly verified from (6.39). The conclusion follows from the standard theory of linear recurrences. The $c_{i}$ 's may be computed from the initial conditions.

Proof of Theorem E. Since $|\bar{\sigma}|<1,(6.36)$ implies that

$$
N_{r}=N\left(T_{d}(\beta) ; \beta_{r+1}-1\right)=c_{1} \sigma^{r}+O(1) .
$$

For any $n$, choose $r$ such that $n \in\left[\left[\beta_{r}-1, \beta_{r+1}-1\right]\right]$, then

$$
N_{r} \leq N\left(T_{d}(\beta) ; n\right) \leq N_{r+1} .
$$

Since $\beta_{n} \approx \beta^{n}$, it follows that $N\left(T_{d}(\beta) ; n\right)=O\left(n^{\log \sigma / \log \beta}\right)=O\left(n^{e(d)}\right)$. Hence $N\left(S_{d}(\beta) ; n\right)=n-o(n)$, so $D\left(S_{d}(\beta)\right)=1$.

Observe that $T_{d}(\beta)$ contains no integers between $\beta_{r+3}$ and $\beta_{r+3}+\beta_{r+1}-1$, as $\varepsilon_{r+2}(m) \geq 1$ and $\varepsilon_{r+1}(m)=\varepsilon_{r}(m)=0$ for $m$ in this interval. Since $\beta_{r+3}+\beta_{r+1}-1 \approx\left(1+\beta^{-2}\right) \beta_{r+3}, n^{-e(d)} N\left(T_{d}(\beta) ; n\right)$ does not converge as $n \rightarrow \infty$.

\section{Discussion of Conjecture F}

One can imagine the method of the last section extended to a slightly larger set of $\alpha$ 's. Suppose $\gamma \in[1, d] \backslash I_{d}$ is a PV number with minimal polynomial $H(x)=x^{k}-\sum c_{i} x^{i}$, where $c_{i} \in[[0, d-1]]$. Then, as before, $a_{n}=\sum \gamma_{j}^{n}$ satisfies the recurrence induced by $H$, and there ought to be a close relationship between $a_{n}$ and $\gamma_{n}=\left[\gamma^{n}\right]$. We now argue probabilistically that $T_{d}(\gamma)$ "ought" to have density zero. If $\gamma<d$, then there exists $r$ such that, for all $s$, $(d-1)\left(\gamma_{s+r}+\cdots+\gamma_{s}\right)>\gamma_{s+r+1}$. Thus, if $\varepsilon_{i}(m)=0$ for $i \in[[s, s+r]]$ and 
$\varepsilon_{s+r+1} \geq 1$, then we may "cash in" $\gamma_{s+r+1}$ for smaller $\gamma_{i}$ 's, as in the last section, and in the ensuing rearrangement, the new $u$ might lie in $S_{d}(\gamma)$. Since the probability that a string of length $r$ contains a fixed pattern increases to 1 as $r \rightarrow \infty$, this argument gives a reason to believe that $D\left(T_{d}(\gamma)\right)=0$.

As $\alpha$ gets close to $d$, the cutoff value $t$ in the greedy representation increases; the explicit description of the strings $(\underline{\varepsilon}(m), u(m))$ seems to become a very difficult problem. However, if $\alpha=d-\delta$, where $\delta>0$ is small, and $r$ is sufficiently small, then we can determine both $S_{d}^{r}(\alpha)$ and $N_{r}$.

Lemma 7.1. If $\alpha \in\left[\left(d^{s}-1\right)^{1 / s}, d\right)$, then $\alpha_{i}=d^{i}-1$ for $1 \leq i \leq s$.

Proof. Certainly $\alpha^{i}<d^{i}$; on the other hand,

$$
\left(d^{i}-1\right)^{1 / i}=d\left(1-d^{-i}\right)^{1 / i} \leq d\left(1-d^{-s}\right)^{1 / i} \leq d\left(1-d^{-s}\right)^{1 / s} \leq \alpha
$$

For fixed $d, t\left(\left(d^{s}-1\right)^{1 / s}, d\right) \approx s-1+\log _{d}(s(d-1))$.

Lemma 7.3. If $r \geq 2$ and $\alpha \in\left[\left(d^{r}-1\right)^{1 / r}, d\right)$, then $N\left(T_{d}(\alpha) ; d^{r}-1\right)=d^{r-1}-$ $d^{r-2}$.

Proof. Suppose $m \leq d^{r}-1$ and $m=\sum_{i=0}^{\infty} \varepsilon_{i} \alpha_{i} ; d^{r}-1=\alpha_{r} \in S_{d}(\alpha)$, otherwise, $\varepsilon_{i}=0$ for $i \geq r$. There are $d^{r}$ formally distinct sums $\sum_{i=0}^{r-1} \varepsilon_{i} \alpha_{i}$, and any such sum is bounded above by

$$
(d-1) \sum_{i=0}^{r-1} \alpha_{i}=(d-1)\left(1+\sum_{i=1}^{r-1}\left(d^{i}-1\right)\right)=d^{r}-(r-1) d+r-2 \leq d^{r}-2
$$

It follows that $N\left(T_{d}(\alpha) ; d^{r}-1\right)=d^{r}-N\left(S_{d}(\alpha) ; d^{r}-1\right)$ equals the number of "repeats" among the sums. Suppose

$$
\sum_{i=0}^{r-1} \varepsilon_{i} \alpha_{i}=\sum_{i=0}^{r-1} \eta_{i} \alpha_{i}
$$

and $\varepsilon_{k}=\eta_{k}$ for $k>j$, but $\varepsilon_{j}>\eta_{j}$; clearly, $j \geq 1$. By pruning common higher terms, we obtain

$$
\left(\varepsilon_{j}-\eta_{j}\right) \alpha_{j}=\sum_{i=0}^{j-1}\left(\eta_{i}-\varepsilon_{i}\right) \alpha_{i}
$$

If $j \geq 2$, then, as before,

$$
d^{j}-1=\alpha_{j} \leq\left(\varepsilon_{j}-\eta_{j}\right) \alpha_{j} \leq(d-1) \sum_{i=0}^{j-1} \alpha_{i}=d^{j}-(j-1) d+(j-2),
$$

hence $(j-1) d \leq(j-1)$, a contradiction. If $j=1$, then (7.6) implies that $\left(\varepsilon_{1}-\eta_{1}\right) \alpha_{1}=\left(\eta_{0}-\varepsilon_{0}\right) \alpha_{0}$, hence $\varepsilon_{1}-\eta_{1}=1$ and $\eta_{0}-\varepsilon_{0}=d-1$. On restoring the pruned terms, we see that (7.5) occurs with $\underline{\varepsilon} \neq \underline{\eta}$ if and only if

$$
\underline{\varepsilon}=\left(\varepsilon_{r-1}, \ldots, \varepsilon_{2}, 1+\varepsilon_{1}, 0\right) \text { and } \underline{\eta}=\left(\varepsilon_{r-1}, \ldots, \varepsilon_{2}, \varepsilon_{1}, d-1\right) \text {, }
$$


where $\varepsilon_{1} \in[[0, d-2]]$ and $\varepsilon_{i} \in[[0, d-1]]$ for $i \geq 2$. There are $d^{r-2}(d-1)$ such pairs, and this completes the proof.

This result shows that we cannot have a uniform bound of the form

$$
N\left(T_{d}(\alpha), n\right) \leq f(d, n)
$$

for all $\alpha \in[1, d] \backslash I_{d}$, where $f(d, n)=o(n)$. Of course, every fixed $\alpha$ is eventually less than $\left(d^{s}-1\right)^{1 / s}$ as $s \rightarrow \infty$.

Taking a broader view, we let $R(\alpha, d, m)$ denote the number of ways that $m$ can be written in the form $m=\sum_{i=0}^{\infty} \varepsilon_{i}\left[\alpha^{i}\right], \varepsilon_{i} \in[[0, d-1]]$. This paper has determined those $(\alpha, d)$ for which $R(\alpha, d, m) \geq 1$ for all $m$. For fixed $(\alpha, d)$, we have an infinite product formula for the generating function:

$$
\sum_{m=0}^{\infty} R(\alpha, d, m) z^{m}=\prod_{i=0}^{\infty}\left(\sum_{k=0}^{d-1} z^{k \alpha_{i}}\right)=\prod_{i=0}^{\infty} \frac{\left(1-z^{d \alpha_{i}}\right)}{\left(1-z^{\alpha_{i}}\right)}
$$

When $\alpha \notin \mathbf{Z}$, the irregularity of $\left(\alpha_{i}\right)$ renders (7.9) unhelpful in understanding $R(\alpha, d, m)$. However, when $\alpha$ is an integer, cancellation can occur.

$$
\sum_{m=0}^{\infty} R\left(k, k^{r}, m\right) z^{m}=\prod_{i=0}^{\infty} \frac{\left(1-z^{k^{r+i}}\right)}{\left(1-z^{k^{i}}\right)}=\prod_{i=0}^{r-1} \frac{1}{\left(1-z^{k^{i}}\right)} .
$$

If $r=2,(7.10)$ leads to the explicit closed formula: $R\left(k, k^{2}, m\right)=[m / k]+$ 1. The computation of $R(2,4, m)$ was Problem B-2 on the 1983 Putnam Competition, see [12].

If $\alpha=2$ and $d=3$, then (7.9) becomes

$$
\sum_{m=0}^{\infty} R(2,3, m) z^{m}=\prod_{i=0}^{\infty}\left(1+z^{2^{i}}+z^{2^{i+1}}\right) .
$$

Let $s(n)$ denote the Stern diatomic sequence (see, $[6,13,15])$, which is defined recursively by:

$$
\begin{gathered}
s(0)=0, \quad s(1)=1, \\
s(2 n)=s(n), \quad s(2 n+1)=s(n)+s(n+1), \quad n \geq 1 .
\end{gathered}
$$

An easy argument shows that $R(2,3, m)=s(m+1)$. The Stern sequence has had a rather fugitive history; see [17]. The exact value of $s(n)$ can be calculated from the pattern of 0 's and 1's in the binary expansion of $n$, as the denominator of a certain continued fraction.

It is easy to see from (7.12) that $s\left(2^{n}\right)=1$ for all $n$; it was already known in the 19th century that the maximum value of $s(m)$ for $m \in\left[\left[2^{n}, 2^{n+1}\right]\right]$ is $F_{n+2}$, the $(n+2)$ nd Fibonacci number (and so is $\left.\approx c \Phi^{n}\right)$. This maximum is achieved twice in each such interval, at the integers closest to $\frac{4}{3} 2^{n}$ and $\frac{5}{3} 2^{n}$. We show in [18] that the growth of $R(2, k, m)$ depends on the parity of $k$ mod 2. There exist positive $c_{i}=c_{i}(k)$ such that, for all $m$,

$$
c_{1} \leq m^{-(\log k) /(\log 2)} R(2,2 k, m) \leq c_{2} \text {, }
$$


but $m^{-\tau} R(2,2 k+1, m)$ cannot be bounded in this way for any $\tau$.

Finally, consider $\lim _{d \rightarrow \infty} R(k, d, m)=R(k, m)$, the number of partitions of $m$ into powers of $k ; R(2, m)$, usually written $b(m)$, was studied by Euler. The asymptotics of $R(k, m)$ have been studied by Mahler [16] and de Bruijn [3]. Mahler proved that, for fixed $k$,

$$
(2 \log k)(\log m)^{-2} \log R(k, m) \rightarrow 1 \text {. }
$$

In a beautiful analysis, deBruijn gave $\log R(k, m)$ up to $o(1)$. Let $h=m / k$, then

$$
\begin{gathered}
\log R(k, m)=\frac{1}{2 \log k}\left(\log \left(\frac{h}{\log h}\right)\right)^{2}+\left(\frac{1}{2}+\frac{1+\log \log k}{\log k}\right) \log h \\
-\left(1+\frac{\log \log k}{\log k}\right) \log \log h+\psi\left(\frac{\log h-\log \log h}{\log k}\right)+o(1),
\end{gathered}
$$

where $\psi$ is a certain periodic function with period one.

\section{BIBLIOGRAPHY}

1. H. L. Alder, The number system in more general scales, Math. Mag. 35 (1962), 145-151.

2. J. L. Brown, Jr., Note on complete sequences of integers, Amer. Math. Monthly 68 (1961), 557-560.

3. N. G. deBruijn, On Mahler's partition problem, Indag. Math. 10 (1948), 210-220.

4. A. S. Fraenkel, Systems of numeration, Amer. Math. Monthly 92 (1985), 105-114.

5. J. A. Fridy, A generalization of $n$-scale number representation, Amer. Math. Monthly 72 (1965), 851-855.

6. C. Giuli and R. Giuli, A primer on Stern's diatomic sequence, Fibonacci Quart. 17 (1979), 103-108, 246-248, 318-320.

7. R. Graham, On a conjecture of Erdös in additive number theory, Acta Arith. 10 (1964), 63-70.

8. _ On sums of integers taken from a fixed sequence, Proceedings Washington State University Conference on Number Theory, March 1971 (J. H. Jordan and W. A. Webb, eds.), Pullman, Wash., 1971, pp. 22-40.

9. R. K. Guy, Reviews in number theory 1973-1983, 6 vols., Amer. Math. Soc., Providence, R.I., 1984.

10. V. E. Hoggatt and C. King, Problem E 1424, Amer. Math. Monthly 67 (1960), 593.

11. __ Solution to Problem E 1424, Amer. Math. Monthly 68 (1961), 179-180.

12. L. F. Klosinski, G. L. Alexanderson and A. P. Hillman, The William Lowell Putnam mathematical competition. Amer. Math. Monthly 91 (1984), 487-495.

13. D. H. Lehmer, On Stern's diatomic series, Amer. Math. Monthly 36 (1929), 59-67.

14. W. J. LeVeque, Reviews in number theory, 1940-1972, 6 vols., Amer. Math. Soc., Providence, R.I., 1973.

15. D. A. Lind, An extension of Stern's diatomic sequence, Duke Math. J. 36 (1969), 55-60.

16. K. Mahler, On a special functional equation, J. London Math. Soc. 15 (1940), 115-123.

17. B. Reznick, A natural history of the Stern sequence (in preparation).

18. _ Restricted binary partitions (in preparation).

Department of Mathematics, California Institute of Technology, Pasadena, CaliFORNIA 91125

Department of Mathematics, University of Illinois at Urbana-Champaign, Urbana, ILLINOIS 61801 (Current address) 